\title{
ULTRA-SHORT BUNCHES BY USING A QUASI-CONTINUOUS COMPRESSOR SCHEME IN A LONG BEAM TRANSFER LINE
}

\author{
H. Schlarb and R. Brinkmann, DESY, Notkestr.85, D-22603 Hamburg, Germany
}

\begin{abstract}
Integration of an X-Ray Free Electron Laser facility is planned in the TESLA design for a future $\mathrm{e}^{+} \mathrm{e}^{-}$Linear Collider. In the present scheme, the FEL drive beam is transported to a central user facility in a several $\mathrm{km}$ long beamline. In this paper we present how the longitudinal wakefield effect for short bunches in combination with a special beamline magnetic lattice can be used to obtain compression down to an rms-bunch length in the range of a few $\mu \mathrm{m}$. Peak current of more than $50 \mathrm{kA}$ would enable the $\mathrm{X}$-Ray FEL facility to generate photon wave lengths in the sub-Angstrom regime.
\end{abstract}

\section{DESIGN OF THE TRANSFER LINE}

In the current design for a future Tera Electron Volt Super Conducting Linear Accelerator (TESLA) the drive beam for the X-Ray FEL is compressed to a bunch length of $\sigma_{z}=25 \mu \mathrm{m}$ and accelerated in the LINAC to energies of $\mathrm{E}_{0}=15$ to $50 \mathrm{GeV}$. After $3 \mathrm{~km}$ the beam is kicked into a $12 \mathrm{~km}$ long transfer line and transported to the central user facility, close to the high energy physics laboratories. An intensive study of wakefields, excited in resistive beam pipes and in the TESLA acceleration modules, opens up the possibility to start with an initial bunch length of $50 \mu$ mand end with rms-values of $2 \mu \mathrm{m}$. In the design proposed here the bunch compression takes place over several stages using the longitudinal properties of the wakefields in combination with compressor modules situated along the beamline. The stages can be decomposed as follows:

- Pre-compression: using the LINAC wakefields $\left(\sigma_{z}=50 \mu \mathrm{m} \rightarrow 5 \mu \mathrm{m}\right)$

- Over-compression: interchange of the tail and the head of the bunch $\left(\sigma_{z}=5 \mu \mathrm{m} \rightarrow 8 \mu \mathrm{m}\right)$

- Compensation: energy correlation from the LINAC wakefields is eliminated by the resistive wall wakefields $\left(\sigma_{z}=5 \mu \mathrm{m}\right)$

- Correlation: rebuilding of an energy correlation using again the resistive wall wakefields $\left(\sigma_{z}=5 \mu \mathrm{m}\right)$

- Final Compression: using energy correlation of the previous stage $\left(\sigma_{z}=5 \mu \mathrm{m} \rightarrow 2 \mu \mathrm{m}\right)$

In order to simulate the various stages a 1-dimensional numerical code has been written. The initial beam parameters are given in the following table :

\begin{tabular}{||lc|c|c||}
\hline \hline Quantity & & Units & Value \\
\hline Energy & $\mathrm{E}_{0}$ & $\mathrm{GeV}$ & 30.0 \\
\hline Long. Emittance & $\epsilon_{z}$ & $\mathrm{keV} \mathrm{mm}$ & 25.0 \\
\hline Charge & $\mathrm{Q}_{0}$ & $\mathrm{nC}$ & 1.0 \\
\hline Bunch length & $\sigma_{z}(\mathrm{rms})$ & $\mu \mathrm{m}$ & 50.0 \\
\hline Peak current & $\mathrm{I}_{\text {peak }}$ & $\mathrm{kA}$ & 2.4 \\
\hline \hline
\end{tabular}

Following points are considered:

- Random distribution in the long. phase space

- Wakefields ( resistive tube \& LINAC )

- Compaction factor in the compressor modules

- Space charge effects.

\section{COMPRESSOR MODULES}

A special beamline magnetic lattice, $240 \mathrm{~m}$ long, with a closed dispersion trajectory and a tuneable positive momentum compaction ${ }^{1}$ of $\alpha_{c}=1-3 \cdot 10^{-5}$ is used to compress the bunch. One module consists of three dipoles (bendradius $=1.2 \mathrm{~km}$, length $=5,10,5 \mathrm{~m}$ ) and FODO-cell structure $\left(\beta_{x, y}^{a v e r}=20 \mathrm{~m}, 22.5 \mathrm{~m}\right)$.

\section{WAKEFIELDS}

In the present scheme the longitudinal correlation between energy and position inside the bunch is generated by the electromagnetic fields co-travelling with the bunch.

\subsection{Resistive Wall Wakefields}

Wakefields induced in a cylindrically tube of finite conductivity are well understood. Analytical solutions for the delta wake potential in the ultra-relativistic limit, up to any multipole order ${ }^{2}$, have been derived in $[1,2,3]$. It has been shown that the short range wakefields can be decomposed in a high frequency $\left(k_{r}=\sqrt{3} / \zeta_{0}\right)$ strongly damped $\left(\alpha_{r}=1 / \zeta_{0}\right)$ oscillator and an additional diffusion term. The characteristic length $\zeta_{0}$ will be given by

$$
\zeta_{0}=\left(\frac{2 b^{2}}{Z_{0} \sigma_{0}}\right)^{1 / 3}
$$

where $b$ denotes the radius, $\sigma_{0}$ the conductivity of the wall material and $Z_{0}=120 \pi$ the impedance of vacuum. For a stainless steel tube $\sigma_{0}=1.4 \cdot 10^{6} \Omega^{-1} \mathrm{~m}^{-1}$ and a radius of

\footnotetext{
${ }^{1}$ Definition of $\alpha_{c}$ is given by $\triangle L / L \equiv \alpha_{c} \triangle E / E$ where $L$ denotes the length of the module and $E$ the energy of the particles.

${ }^{2}$ In the following only the monopole term is considered. The higher orders $(n>0)$ inherent by the fraction $(r / b)^{2 n}$ where $r$ denotes the transverse off-set of the beam from the center of the pipe. They can be neglected for $(r \ll b)$.
} 
$b=5 \mathrm{~cm}$ the characteristic length is $\zeta_{0}=211 \mu \mathrm{m}$. For two point-like particles the longitudinal monopole wake potential reads ${ }^{34}[2]$

$$
\begin{aligned}
W_{0, \|}(\zeta)=-\frac{Z_{0} c}{\pi b^{2}}\left(\frac{4}{3} e^{-\alpha_{r} \zeta} \cos \left(k_{r} \zeta\right)\right. & \\
& \left.-\frac{12 \sqrt{2}}{3 \pi} \int_{0}^{\infty} d x \frac{x^{2} e^{-x^{2} \alpha_{r} \zeta}}{x^{6}+8}\right)
\end{aligned}
$$

with $\zeta$ the distance between the source charge and the test charge. For velocities $v$ smaller than the speed of light $c$ the effective bunch length at the wall and the retarded effect of the 'radiated' electromagnetic fields produced by the image current modify the delta wake potential for very short distances $|\zeta|<b / \gamma$, where $\gamma$ denotes the Lorentz factor. The longitudinal delta wake potential per unit length as a function of $\zeta$ is shown in Fig.1.

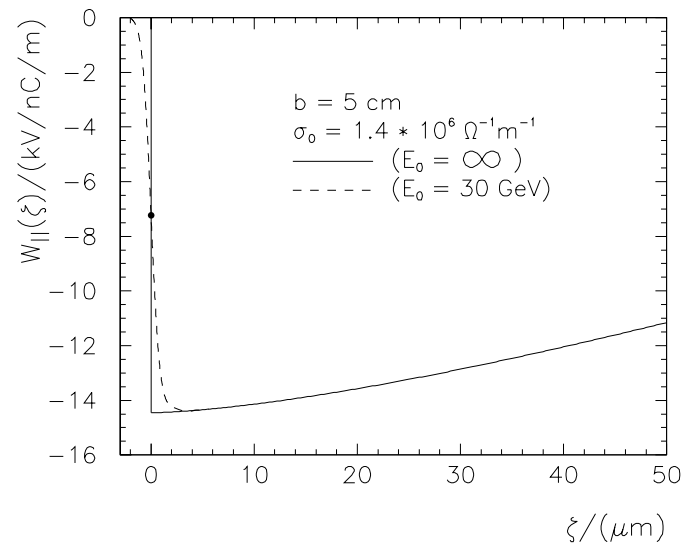

Figure 1: Delta wake potential

For distances in the range $b / \gamma<\zeta \ll \zeta_{0}$ the short range wakefields act purely capacitive and the forces are independent of $\zeta$. The wake potential for a charge distribution with a rms-bunch length $\sigma_{z}$ in the above range will be given by integration of the charge longitudinally. In the special case of a Gaussian charge distribution the longitudinal wake potential will be approximately given by the error-function (see Fig.2).

The energy correlation produced by the bunch due to the resistive wall wakefields is linear at the center of the bunch and obeys automatically the desired phase relation. This behavior of self-regulated correlation can not be produced by an off-crest phase acceleration of the beam in a LINAC. For very short bunch lengths an off-crest acceleration will fail anyway since the amplitude of the generated wakefields grows quadratically with the resonance frequency of the cavities $\left(W_{\|} \propto f_{r e s}^{2}\right)$, while the resulting compensation will only be proportional to the $f_{\text {res }}\left(d E / d z \propto f_{\text {res }}\right)$.

\footnotetext{
${ }^{3}$ The dc-model of conductivity has been used. In case of $\zeta_{0} \approx \tau c$ where $\tau$ denotes the relaxation time of the conduction electron $(\tau \sim$ $\left.10^{-15} s^{-1}\right)$ the frequency-dependent Ohm's law has to be used. For ourer parameter set it can be neglected.

${ }^{4}$ The anomalous skin effect has not been taken into account. The assumption is justified for materials of poor conductivity if the penetrationdepth $\delta=\sqrt{2 c / \omega_{c} Z_{0} \sigma_{0}}$ at the cut-off frequency $\omega_{c}=b / \gamma c$ is much larger than the mean free-path $l=v_{F} \tau$ of the conducting electrons.
}

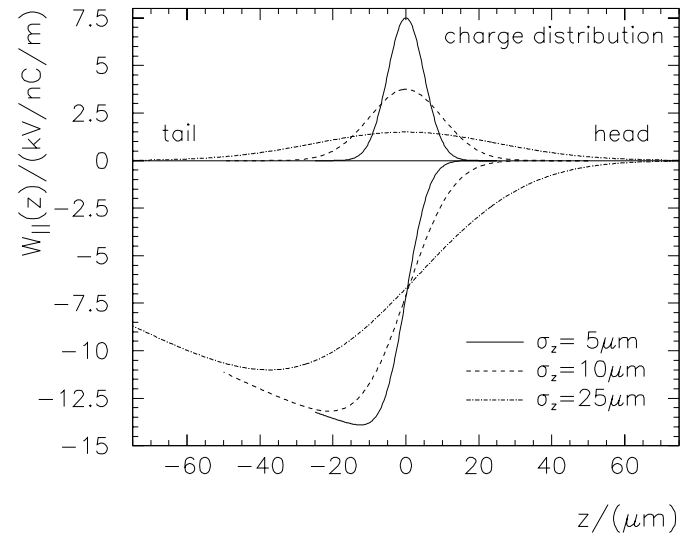

Figure 2: Wake potential for Gaussian charge distr.

\subsection{Wakefields of the TESLA Module}

The longitudinal wakefields for one TESLA module, $10.4 \mathrm{~m}$ long, consisting of 8 cavities has been calculated [4]. The results are shown in Fig.3 together with the wake produced by the 8 th cavity normalized to one module. It can be seen that both wake potentials can be used for a precompression of the bunch coming from the LINAC. The latter, being more linear over the bunch, would lead to a better performance. Since the wakefields for several modules are not yet available the average wake potential (solid curve) is used for numerical simulations. We expect that the LINAC-wakefields of 250 modules $(3 \mathrm{~km})$ will look more like the wake of the 8 th cavity (dashed curve). This would increase the efficiency of the compressor scheme by $30 \%$.

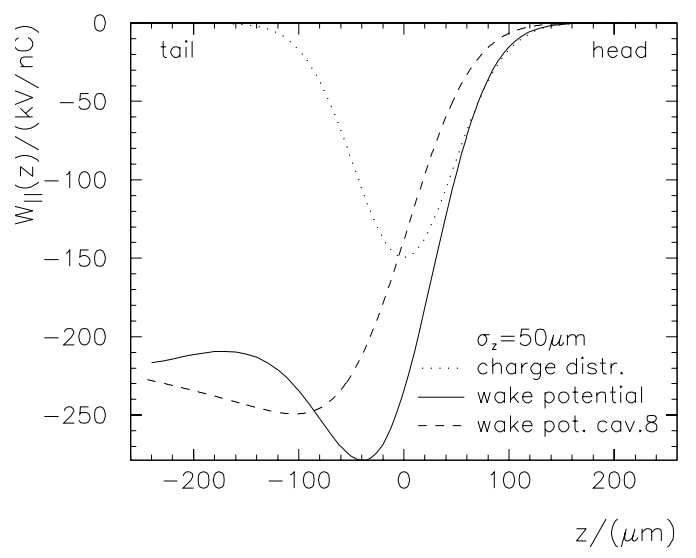

Figure 3: Wake potential of one TESLA module

\section{RESULTS}

\subsection{Longitudinal Charge and Energy Distribution}

The nonlinearities of the wake potentials at the tails of the bunch reduces the efficiency of such a method. The present simulation shows that approximately $50 \%$ of the initial charge can be compressed to extremely small values of less than $2 \mu \mathrm{m}$ shown in Fig.4(a). The energy distribution of the core of the bunch is shown in Fig.4(b) and reflects essentially the longitudinal emittance of the initial bunch. The two typical peaks at the edges of the energy dis- 
tribution are caused by the over-compression in the second stage. This stage is mainly used to increase the efficiency and the stability of the compressor scheme. Note that the LINAC can run with an on-crest acceleration phase, since no compensation of the wakefields is needed.
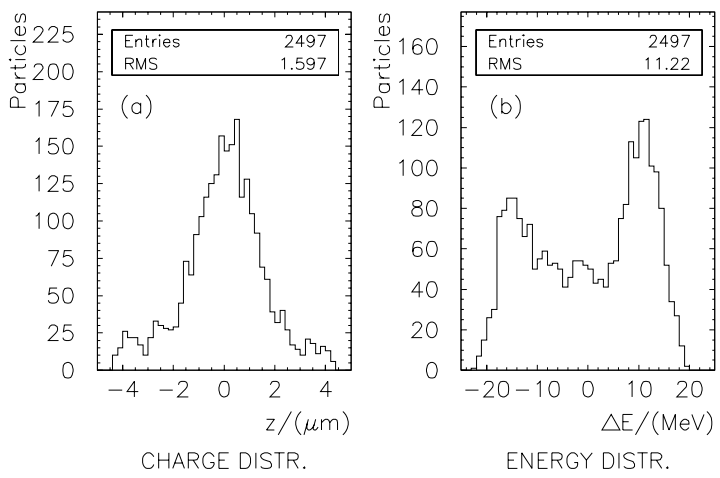

Figure 4: Longitudinal charge distribution (a) and energy distribution (b) for $50 \%$ of the initial charge $Q_{0}$; the peak current reach $I_{\text {peak }}=50 \mathrm{kA}$.

Finally the bunch in the longitudinal phase space at the end of the transfer line is shown in Fig.5. The tails of the bunch have to be removed by collimation.

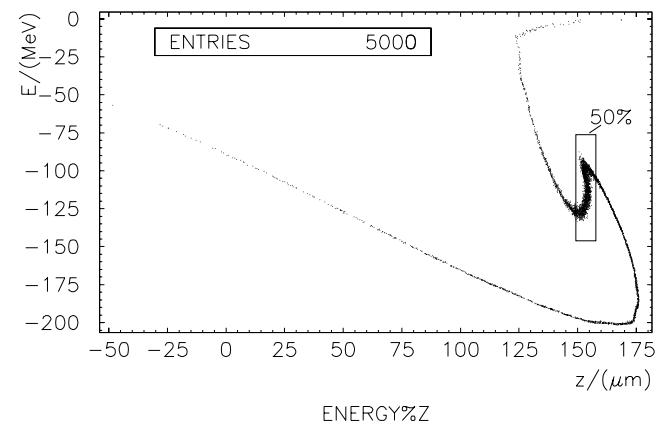

Figure 5: Longitudinal phase space distribution of the particles at the end of the compression scheme; $\mathrm{E}=0$ corresponds to the initial beam energy $\mathrm{E}_{0}=30 \mathrm{GeV}$.

\subsection{Stability of the Compression scheme}

An important question is the stability of such a scheme for different initial bunch parameters since the energy correlation is generated by the bunch itself. This has been studied in detail for bunch to bunch charge fluctuations up to $\pm 20 \%$ while the other parameters have been kept constant. The results are given in Fig.6(a) and (b). The variation is limited to reasonable values for the bunch length, the peak current and the energy spread.

\section{OPEN QUESTIONS}

The presented values for the peak current are orders above any existing and planned machine so that we expect additional limiting effects for the practical realization of such a compression method. One of these is the coherent synchrotron radiation (CSR)[5] generated in the bending system of the compressor modules for a bunched beam. This
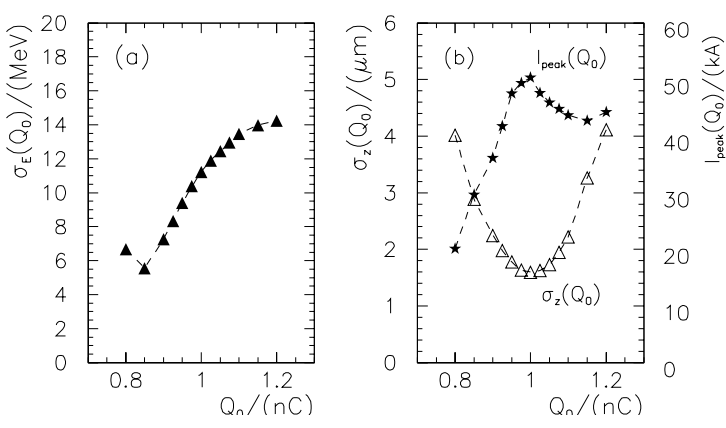

Figure 6: The rms-energy distribution (a) and the rmsbunch length together with peak current (b) for $50 \%$ of the initial charge $Q_{0}$ as a function of $Q_{0}$.

tail-head effect may lead to a coupling between the coherent energy spread generated by the CSR and the transverse (bending) plane coordinates through the chromatic transfer function of the particular beamline. This effect is under study and first estimations shows that this can be the limiting effect for our scheme. It is in principle possible to cancel this effect [6], but the final bunch length will be limited to values somewhat larger than shown above.

\section{CONCLUSION}

We have presented how the longitudinal wakefield effect can be used to obtain bunch lengths below $2 \mu \mathrm{m}$ and peak currents above $50 \mathrm{kA}$ with an efficiency of $50 \%$. The concept of over-compression lead to a first order compensation of bunch to bunch charge fluctuations and guarantee the stability of the quasi-continuous compressor scheme up to $\triangle Q_{0} \approx \pm 10 \%$.

\section{ACKNOWLEDGEMENTS}

One of the author wishes to thank A. Novokhatski and A. Piwinski for the helpful discussions about the properties of wakefields.

\section{REFERENCES}

[1] O.Henry \& O.Napoly, "The Resistive-Pipe Wake Potentials for Short Bunches", Part. Acc., Vol. 35, pp. 235-247,1991

[2] K. Bane, "The Short Range Resistive Wall Wakefields", SLAC-AP-87, (June 1991)

[3] K. Yokoya, "Resistive Wall Impedance of Beam Pipes of General Cross Section", Part. Acc., Vol. 41, pp. 221-248,1993

[4] A. Novokhatski \& A. Mosnier, "Short Range Potentials for a Chain of TESLA cavities", DAPNIA/SEA-96-08, (Nov. 1996)

[5] Ya. S. Derbenev et. al., "Microbunch Radiative Tail-Head Interaction", DESY, (Sep. 1995)

[6] P. Emma \& R. Brinkmann, "Emittance Dilution Through Coherent Energy Spread Generation in Bending Systems", contribution to this conference 